\title{
Author Correction: Reclassifying neurodegenerative diseases
}

Pablo Villoslada, Ricardo Baeza-Yates and Joseph C. Masdeu

Correction to Nature Biomedical Engineering https://doi.org/10.1038/s41551-020-0600-3, published online 03 August 2020.

In the version of this News \& Views originally published, author Ricardo Baeza-Yates' first affiliation was misspelt as 'Khory College of Computer Sciences, Northeastern University at Silicon Valley, San Jose, CA, USA'; it should have read 'Khoury College of Computer Sciences, Northeastern University at Silicon Valley, San Jose, CA, USA'. This has now been corrected.

Published online: 17 September 2020

https://doi.org/10.1038/s41551-020-00628-2

๑) Springer Nature Limited 2020 